

RESEARCH ARTICLE

\title{
THERMO-ELECTRICAL CHARACTERIZATION OF SMART BIO COMPOSITE MATERIAL (PLA-G-
} CF)

\section{Mohammed Basheer E.P ${ }^{1}$ and Dr. K. Marimuthu ${ }^{2}$}

1. Research Scholar Advanced Manufacturing Technology, Coimbatore Institute of Technology, Anna University, Tamil Nadu, India.

2. Professor and Head of the Department, Department of Mechanical Engineering, Coimbatore Institute of Technology, Anna University, Tamil Nadu, India.

\section{Manuscript Info}

Manuscript History

Received: 10 February 2020

Final Accepted: 12 March 2020

Published: April 2020

Key words:-

Additive Manufacturing, Biodegradable

Polymer, PLA, Graphene (G), Carbon

Fiber (CF), Composite, Filament, FDM
Abstract

Biodegradable and Bio-compactable characteristics of Polylactic Acid (PLA) attracted the attention for numerous biomedical applications. Numerous tissue engineering problems, restoration of weakened or damaged tissues have been reported by using PLA and its copolymers due to their biocompatibility and distinctive mechanical and electronic properties. Similarly, biomedical application of Graphene and Carbon fiber can be exposed with significant potential. Therefore, use of graphene and carbon fiber blend to PLA can positively impact, accelerate and can provide very high influence in tissue engineering, regenerative medicine and other biomedical sectors. In this research study, a screw extruder is used to synthesis PLA-G-CF polymer composite filaments of various nano and micro particles with different particle morphology as reinforcement to PLA matrices. Polylactic Acid (PLA) has been used as matrix material and Graphene and Carbon fiber particles used as reinforcement. These polymer composite filaments are then used for Additive Manufacturing to fabricate medical components. The aim of this work was to investigate a potential method of preparing and processing biodegradable Polylactic Acid (PLA), Graphene and Carbon fiber (CF) through 3D Printing (FDM) Technology. In this research, the capability to use materials with conductive properties are also investigated which may lead to a new generation of 3D smart material. Synthesized composite filaments were characterized through Scanning Electron Microscope (SEM), Differential Scanning Calorimetry (DSC), Tensile Test and Fatigue Test. Among the various blend proportions considered in this work, 80/20\% PLA-G-CF blend exhibited the highest elongation and impact strength. Diffe-rential Scanning Calorimetry (DSC) showed the influence of Graphene and Carbon fiber content on glass transition temperature ( $\mathrm{Tg}$ ), melting temperature and degree of crystalline of PLA-G-CF blends. Based on their characterization properties, PLA-G-CF Composite material can focus on various biomedical applications such as scaffolds, implants, drug delivery, cancer therapy, biological imaging etc.

Copy Right, IJAR, 2020,. All rights reserved. 


\section{Introduction:-}

Today, the environmental pollution has become a great concern and evil due to the high impact of plastic wastes in daily use. One of the possible solutions to this problem is to replace the commodity synthetic polymers with a biodegradable and ecco-polymers which are readily susceptible to microbial action. Among them, bio-based and biode-gradable polymers, Polylactic Acid (PLA) has been attracting much attention due to its mechanical properties resembling that of present day commodity plastics such as PE, PP and PS. Pure PLA is usually colorless and glossy thermoplastic polymer with similar properties as that of polystyrene. It can be processed using injection-molding, compres-sion-molding, extrusion, thermoforming etc (Garlotta et al., 2002). Polylactic acid (PLA) polymers are synthesized and obtained from renewable agricultural resources by polymerization of lactide, which is the cyclic diester of lactic acid (Milena et al., 2014). PLA has high modulus, reasonable strength, excellent flavor and aroma barrier capability, good heat seal ability and can be readily fabricated,

The tensile strength gained from PLA structures ranges from 15.5 MPa to 89.1 MP. There by making it one of the most promising biopolymers for varied applications.PLA polymers have already been demonstrated as surgical implant materials, drug delivery systems (Rancan et al., 2009 and Saini et al.,2016) guided tissue and bone regeneration platforms, and also as porous scaffolds for the growth of neo-tissue (Wang et al, 2016 and Liakos et al, 2016). Use of PLA in biomedical applications is not based only on its biodegradability but also on its thermomechanical properties (Tcharkhtchi et al., 2014; Saini et al., 2016 and Hamad et al., 2015). Hence, various medical devices have been prepared from different PLA types including degradable sutures, drug releasing micro-scale particles, nano-particles, and porous scaffolds for cellular applications (Rasal et al.,2016).Despite these desirable features, several drawbacks tend to limit its widespread applicability such as high cost, lower mechanical properties, and narrow processing windows (Dedenaro et al., 2016). Thus in order to broaden the applications of PLA, material properties and process ability has to be improved by blending with some higher grade biocompatible material. Even though, PLA polymers have a numerous limitations, significantly, thermal and mechanical properties, its tensile strength and elastic modulus are comparable, but poor toughness limits its use in applications at higher stress levels. For instance, when Polylactic acid interference screws were used for graft fixation during anteriorcruciate ligament (ACL) reconstruction, the devices were reported to be mechanically weaker than their metallic counterparts and often fractured during implantation (Rasal et al.,2016) . Its biodegradation rate is considered to be quite slow, which depends on its crystalline and molecular weight of the material (Dedenaro et al., 2016).

Graphene, on the other hand, has been at the forefront in bio-technological and medical applications (Aumnate et al., 2018; Zhang et al., 2012; Bitounis et al., 2013 and Paul et al., 2011). In particular, the role of its surface chemistry, size, and ability to adsorb active bio-molecules has a huge impact on their biomedical applications (Bitounis et al., 2013). Graphene is a free-standing $2 \mathrm{D}$ crystalline structure with one-atom thickness. It is an al-lotrope of carbon comprising layers of six-atom rings in a honeycombed network and can be theoretically viewed as a true planar aromatic macromolecule (Xianfeng Zhou et al., 2014 and Sithiprumnea Du et al., 2016). Graphene exhibits remarkable properties (Wang et al., 2011) such as unusual electronic flexibility as well as high planar surface area $(\sim 2630 \mathrm{~m} 2 / \mathrm{g})$, outstanding mechanical strength (Young's modulus, $\sim 1100 \mathrm{GPa}$ ) and unparalleled thermal conductivity $(\sim 5000 \mathrm{~W} / \mathrm{m} / \mathrm{K})$. As such, it is a highly popular nano-scale additive for reinforced biopolymers (Scaffaro et al., 2016). Bio-safety of graphene and graphene derivatives is attracting more attention as it enters into a biological system (Wang et al., 2011; Scaffaro et al., 2016 and He Shen et al., 2012). It has been acknowledged that the biological effect of graphene is largely affected and complex by the exposure dose, methods, chemical function, lateral size, thickness, and surface properties (Yong Cheol Shin et al., 2017 and Shen et al., 2016). Both graphene and graphene derivatives can be adapted to function as smart biomedical devices as long as their surfaces are properly functionalized (Zhang et al., 2016). Moreover, (Dul et al., 2016) successfully prepared the ABS/GNP composite for the FFF process using the melt compounding method. The presence of GNP led to the reduction in the co-efficient of thermal dilation and improved the stability under long lasting loads for the 3D printed parts.

Polymer blends as composites are the one of the convenient approaches to tailor the ma-terial properties at low processing cost. Polymer blending is a method for obtaining properties that the individual do not possess and has been widely used for various kind polymers. Blending of PLA with other polymers offers the possibility of improving the degradation rate, permeability characteristics, thermal and mechanical properties. The objective of this research is to produce different blends of PLA, Graphene and Carbon fiber and to investigate the mechanical and chemical characterization. The research also reports natural jute fibers with PLA matrix that results in high 
ductility instead of tensile strength, as compared to carbon fibers' reinforced parts. Therefore, the brittle carbon fibers and graphene provide high strength and natural fibers exhibiting low brittleness attain higher ductility. The non-treated carbon fibers and graphene with PLA matrix depicted the highest flexural strength of $335 \mathrm{MPa}$ (Matsuzaki et al., 2016). In Rangisetty and Peel's study, they investigated the mechanical properties of the composite materials produced by FDM process. As filament materials, they used PLA, PLA/CF, ABS, ABS/CF, PETG and PETG/CF filaments from commercial products. As the result of study, rigidity of all the fiber reinforced composites were increased, but surprisingly the tensile strengths were decreased probably due to very short fiber lengths (Rangisetty et al., 2016).

Additive manufacturing (AM) or $3 \mathrm{D}$ printing is the next generation manufacturing tech-nology that allows manufacturing of complex parts without requiring specialized tooling (Hsiao et al., 2018). Additive manufacturing (AM) is a bottom to up process, creating ob-jects directly from material deposition layer by layer in a controlled manner. Because of this unique approach, AM allows to manufacture complex shaped parts with minimum material wastage and energy consumption (Diegel et al., 2006). FDM/FFF is one of the most prominent and prevalent AM Technology due to its devices simplicity and wide availability of materials. In Fused Filament Fabrication, melt thermoplastic polymers are extruded to make the layers for fabricating the design provided in one of the following formats: STL (stereo lithography), AMF (additive manufacturing file), Step (standard for exchange of product model data), Voxel, 3MF (3D manufacturing format), or JT (Jupiter tessellation) (Khoo et al., 2015; Campbell et al., 2012; Umaras et al., 2017 and Loh et al., 2018). Today, ABS, Polycarbonate (PC), Polylactic Acid (PLA), Polyamide (PA), Polystyrene (HIPS), Poly-Caprolactone (PCL), polyvinyl Alcohol (PVA), and Polyurethane (TPU) and the mixtures of different types of thermoplastics are the most common feedstock material in other words filaments in FDM technique (Przybytek et al., 2018; Tsai et al., 2018; Zocca et al., 2018; Khatri et al., 2018 and Hohimer et al., 2018). However, the parts to be manufactured using these filaments lack biodegradability and biocompatibility, due to inherent nature of the polymeric materials, to anti oxidation property, etc. This short coming restricts implementation of the FDM technology significantly and limits its applicability to industrial, medical and domestic applications. This section highlights the tensile capability of various potential FFF (Rumley-Ouellette et al., 2017; Pranzo et al, 2018; Tanikella et al.,2017 and Wu et al., 2015). Therefore, there is an urgent need for development of novel materials with enhanced properties. Biodegradable Polymer nano-composites stand out as a potential solution to overcome this limitation.

\section{Material and Methods:-}

\section{Preparation of PLA Composite Filament:}

PLA is mixed mechanically with reinforcement powder and then is melt compounded using the screw. This method is widely used for preparing thermoplastic nano-composite. For the filament extrusion process, the PLA composite with 1.5 to 2 wt.\% of reinforcement content was mixed to 98 to 98.5 wt.\% of pure PLA granules. This mixture was then melted and extruded using the screw extruder. The extrusion temperatures ranged from $180^{\circ} \mathrm{C}$ to $220^{\circ} \mathrm{C}$ from the hopper to the die. Thus, the $1.75 \mathrm{~mm}$ diameter filament was produced and collected for the 3D printing test. Figure 1 demonstrates the equipment setup for preparing the PLA Composite filament by the melt extrusion process.

\section{Fabrication of PLA-TPU/ABS/G/CF Composite Filaments:}

The process starts feeding the hopper with the PLA and TPU/ABS/Graphene/Carbon fi-ber granules or pellets in a proportionate condition. The screw extrusion pushes and breaks the granules due to the thermal condition provided by the induction coils. The ex-trusion process continues until the material comes out of the nozzle in the form of wire. The screw extrusion is utilized for the filament composite production.

The main part of the extruder is a barrel containing a screw (also sometimes referred to as an "auger", which is connected to a heater towards its far end. On the other end, the screw is connected to an electric motor which will, via mechanical action, transport the so-called resin pellets through the barrel towards the heater. Pellets are gravityfed continuously from a hopper (a kind of feeding funnel). As the motor is continuously driving the auger, the resin pellets are pushed into the heater. The thermoplastic granules will soften and melt because of the heat generated and are then pushed mechanically through a die. Pushing the soft thermoplastics through the die will cause it to form a continuous filament strand with the diameter of the diameter $(1.75 \mathrm{~mm})$. This process is called extrusion, hence the term "extruder". 




Figure 1:- Screw Extrusion Equipment (fused filament extrution system).

Table 1:- The weight ratio of the particles added into the PLA matrix. (Table caption is indispensable).

\begin{tabular}{|l|l|l|l|l|}
\hline \multirow{2}{*}{ S.No. } & \multicolumn{2}{|l|}{ Weight Ratio added to PLA Matrix } & \\
\cline { 2 - 5 } & PLA (wt\%) & Additive (wt $\%)$ & Additives & Extruded Filament \\
\hline 1 & 98.5 & 1.5 & PLA + TPU & \\
\hline 2 & 98.5 & 1.5 & PLA + ABS & \\
\hline 3 & 98 & $\begin{array}{l}2(1 \%-\text { G \& } 1 \%- \\
\text { CF })\end{array}$ & $\begin{array}{l}\text { PLA + Graphene(G) + } \\
\text { Carbon fiber(CF) }\end{array}$ & \\
\hline 4 & 100 & 0 & PLA & \\
\hline
\end{tabular}

\section{Results and Discussion:-}

Characterization of PLA Composite Filaments:

The composite filaments were characterized via different methods, X-Ray Diffraction (XRD) Analysis, DSC, TGA, tensile test, surface roughness, and SEM-EDS.

\section{SEM Microstructure of Fracture Surface:}


Figure 2:- The SEM microstructure of a) PLA FILAMET, b) PLA-G-CF FILAMENT.

The alternative performance of graphene based nano filler-reinforced composites depends on the dispersion and distribution of graphene-based materials in the polymer matrix and the interfacial bonding between these two phases. The microstructures of the fractured sections of PLA-G-CF (dry mixing) on cross section are shown in Figure. PLA-G-CF demonstrated a higher-porous surface with the presence of dispersed Graphene flakes embedded in the PLA matrix. The smaller size and the more uniform distribution of Graphene and Carbon fiber were the critical keys for enhancing the mechanical behavior and for the thermal diffusivity of the composite. 

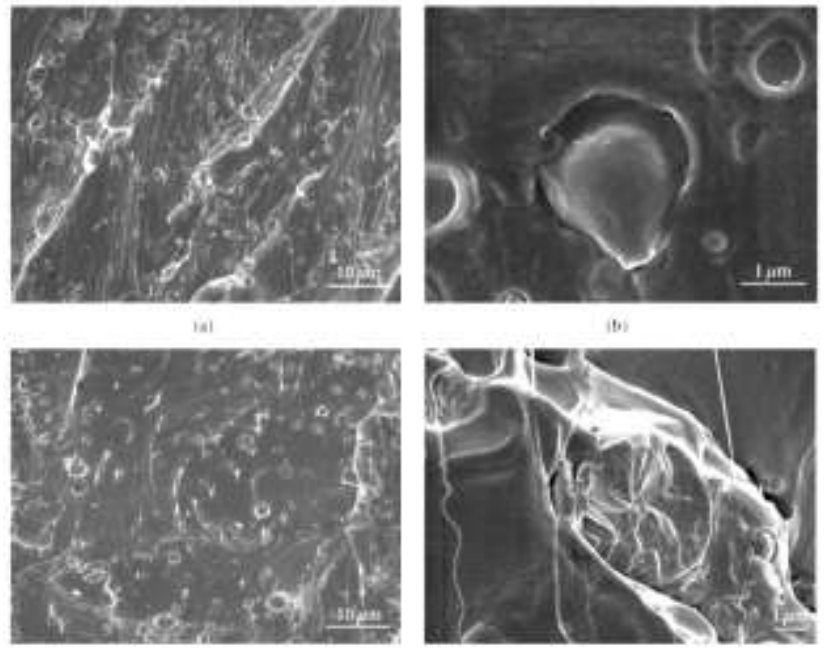

Figure 3:- SEM images of PLA-G-CF dry mixing composite filaments.

\section{DSC Studies of the Produced Filaments:}

Differential scanning calorimetry (DSC) is one of the thermal analysis methods. It is used for measuring thermal flow difference between the sample and the reference material during the exposure of the sample to a controlled temperature and atmosphere. Thermal properties of pure PLA, PLA Flexible, PLA Hard and PLA-G-CF blend were obtained from their DSC thermographs. This study has ability to monitor the transformations in the solid state, phase changes and to determine the thermodynamic parameters during the controlled heating and cooling of the sample.

The samples of the weight of about $10 \mathrm{mg}$ were put in an aluminium bowl, sealed by a press and then placed in a DSC testing device where glass transition temperature, melting temperature, crystallization temperature etc were determined. The parameters that can be determined by DSC analysis are glass transition temperature, crystallization temperature, melting temperature, polymer crystallinity percentage, specific heat capacity, transformation enthalpy and many others. The variation of glass transition temperature, cold crystallization temperature and melting temperature are briefed in the graph given below. For all of the produced filaments, results of the DSC studies were shown in Figure. According to results Pure-PLA granules and PLA-CF-G shows a glass transition temperature (Tg) around of $170.5^{\circ} \mathrm{C}$ and $116^{\circ} \mathrm{C}$.

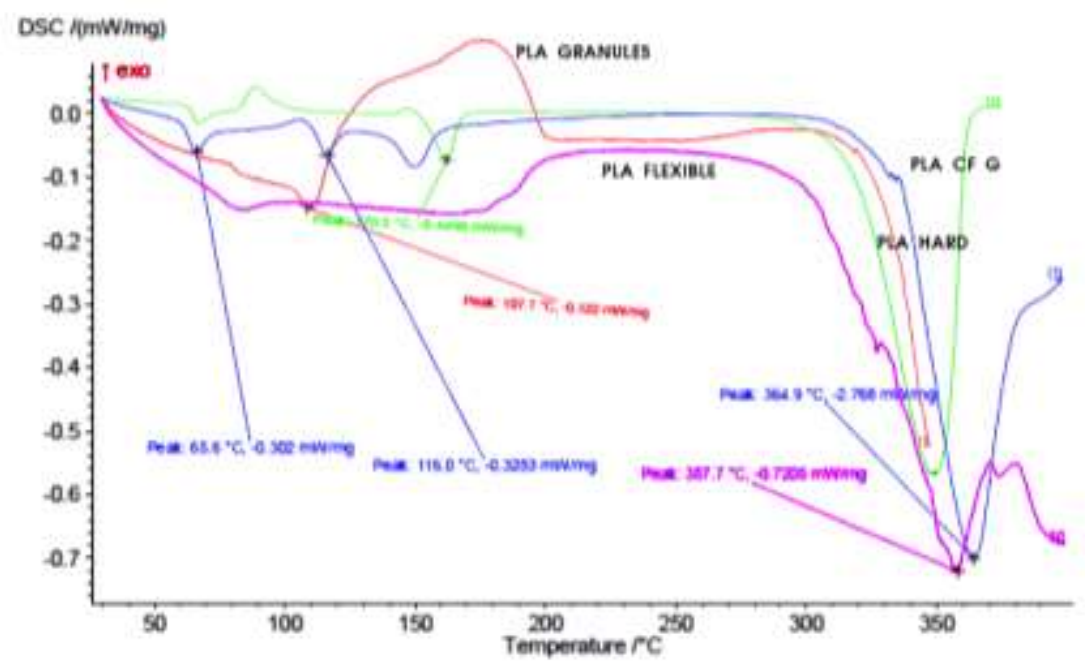

Figure 4:- SEM images of PLA-G-CF dry mixing composite filaments.

It can be seen in Figure 4 that first transition of PLA-CF-G is at $-116{ }^{\circ} \mathrm{C}$ and it represents the glass transition temperature. Next transition is in the interval from 0 to $70{ }^{\circ} \mathrm{C}$ and it represents PLA-CF-G melting. Endothermic 
peak of melting is at $65.6{ }^{\circ} \mathrm{C}$. Third transition is cold crystallization of PLA in the interval from $110{ }^{\circ} \mathrm{C}$ to $364.9^{\circ} \mathrm{C}$. Exothermic peak of cold crystallization is at $300{ }^{\circ} \mathrm{C}$. This result suggests that the addition of Graphene and Carbon fiber to the PLA mixture does not significantly affect the mobility of the polymer in the polymer network. Visible changes are present in the cold crystallization temperature and enthalpy of crystallization for all measured samples. The values are shifted to lower points after addition of higher fibre concentration, which is common for composite materials.

\section{TGA Analysis or Thermo Gravimetry:}

TGA analysis measures the amount of weight change of a material, either as a function of increasing temperature, or isothermally as a function of time, in an atmosphere of nitrogen, helium, air, other gas, or in vacuum. TGA analysis can be interfaced with a mass spectrometer RGA to identify and measure the vapors generated, though there is much greater sensitivity when the mass spectroscopy heating is performed in an ultrahigh vacuum system.

TGA determines temperature and weight change of decomposition reactions, which often allows quantitative composition analysis and also used to determine water content or the residual solvents in a material. This analysis is used as one of the several complementary techniques for identification of an unknown polymer composite content. XPS identified the composition of the reinforcements and revealed the presence of PLA composite as residues of a lower temperature TGA run than the high temperature run shown below. TGA was then performed on the material to find the weight percent of each material. The sample was heated from room temperature to $400^{\circ} \mathrm{C}$ at a rate of $5^{\circ} \mathrm{C} / \mathrm{min}$ in air. The TGA curve (below) is labeled in terms of the identity of the components.

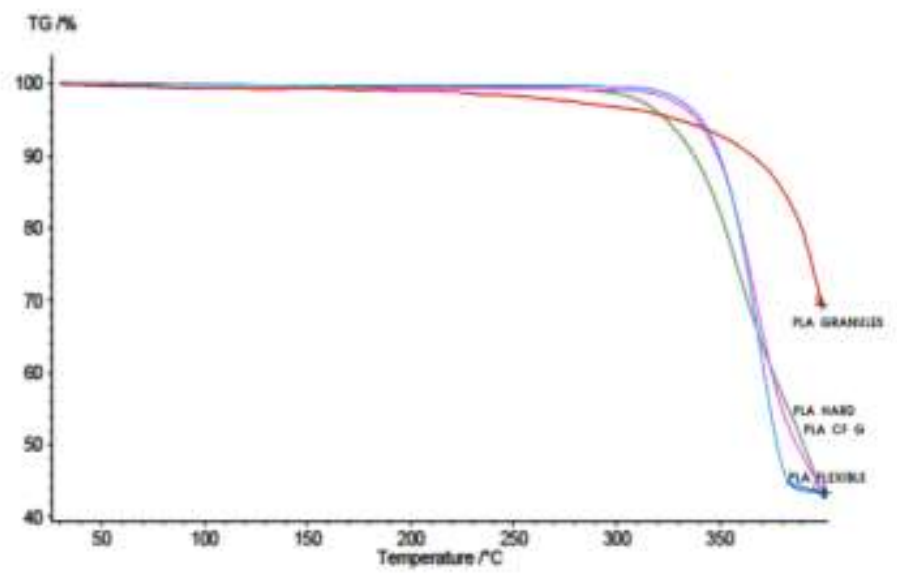

Figure 5:- Determination of weight \% of composite material components by TGA Analysis.

\section{Thermo-Electrical characterization of Smart material:}

The research also concentrates on employing electrical conductive materials in the composites to obtain a smart material. It is possible to 3D print active or passive elements that are printed on their own same materials. In this study, Commercial Polylactic acid (PLA) and FDM represent a benchmark to understand which the real potentialities of smart objects 3D printing are. PLA-G-CF Composite conductive filament has been characterized in terms of resistivity. Then a resistance vs temperature characteristic of this material was attained, which are a new class of low cost materials that promise to substitute metals and semiconductors, opening the way to a new generation of 3D printed smart objects. The results of the conductive PLA-G-CF Composite thermo-electrical characterization indicate that this material is not only exploitable for its electrical conductivity, but also for its significant variation as a function of temperature. Future investigations will be focused on more complex objects in which the PLA-G-CF conductive smart composite in 3D pattern which can be employed for both temperature sensing and electrical pulse sensing.

\section{Conclusion:-}

Fused filament fabrication (FFF) is a promising additive manufacturing (AM) technology due to its ability to build thermoplastics parts with advantages in the design and optimization of models with complex geometries, great design flexibility, recyclability and low material waste. This technique has been extensively used for the manufacturing of conceptual prototypes rather than functional components due to the limited mechanical properties 
of pure thermoplastics parts, new 3D printing filament from PLA-Graphene-Carbon fiber composite was successfully prepared by solution mixing and followed by melted extrusion using a screw extruder. Characterization results shows that these novel composites can be used as filament material in commercial FDM devices without requiring modification. According to the results;

1. The graphene and Carbon fiber reinforced PLA composite filaments for FDM type 3D printer were produced through a twin screw extruder between $190 \mathrm{oC}-220 \mathrm{oC}$ without degradation of the matrix.

2. Thermal properties of neat PLA and PLA-CF-G blends were obtained from their DSC thermographs. DSC studies showed that produced filaments were compatible with commercial 3D printers because of having lower $\mathrm{Tg}$ values. However, a Melt Flow Index (MFI) should be applied on the composite polymers for determining exact processing temperature.

3. The solvent system improved the Graphene and Carbon fiber dispersion capability in the PLA matrix.

4. Graphene and Carbon fiber dispersion improved the thermal properties of the PLA-CF-G composite.

5. SEM microstructure of fracture surfaces indicated PLA-CF-G demonstrated a higher-porous surface with the presence of dispersed Carbon fiber and Graphene flakes embedded in the PLA matrix. The smaller size and the more uniform distribution of Carbon fiber and Graphene were the critical keys for enhancing the mechanical behavior and for the thermal diffusivity of the composite.

6. During the extrusion process, the nano composite filaments need to be fabricated-granulated for a couple cycles to achieve well dispersion of the reinforcement particles in the PLA matrix.

7. Improving mechanical properties verified the feasibility of 3D printed PLA-CF-G for potential use in engineering applications. The tensile strength and Young's modulus of PLA were enhanced naturally by adding Carbon fiber and Graphene. Besides, the elongation at break decreased and needed to be further improved.

Our study demonstrated one of the active attempts to print PLA-CF-G based composite using FFF 3D printing process directly. However, the printed part quality, functionalities, and applications based on the printed PLA-CF-G composite needed to be further exploited.

\section{References:-}

1. Garlotta, D., (2002). A Literature Review Of Poly Lactic Acid, Journal Of Polymers And The Environment, 9(2), Pp. 63-84,.

2. Milena, S., Lopes., Andre, L., Jardiniand Rubens., M. Filho., (2014). Synthesis And Characterizations Of Poly (Lactic Acid) By Ring-Opening Polymerization For Biomedical Applications., Aidic Servizis.R.L., The Italian Association Of Chemical Engineering Doi: 10.3303/Cet1438056, Vol. 38.

3. Rancan, F., Papakostas, D., Hadam, S., Hackbarth, S., Delair, T.; Primard, C.; Verrier, B.; Sterry, W.; BlumePeytav, U.; Vogt, A, (2009). Investigation Of Polylactic Acid (Pla) Nanoparticles As Drug Delivery Systems For Local Dermatotherapy. Pharm. Res., 26, 2027-2036.

4. Saini1, P., Arora, M., Ravi Kumar, M.N.V. (2016).Poly(Lactic Acid) Blends In Biomedical Applications. Advanced Drug Delivery Reviews 107, 47-59

5. Wang, J.,Wang, L., Zhou, Z., Lai, H., Xu, P., Liao, L.,Wei, J. (2016). Biodegradable Polymer Membranes Applied In Guided Bone/Tissue Regeneration: A Review. Polymers, 8, 115.

6. Liakos, L., Grumezescu, A.M., Holban, A.M., Florin., Dautilia, F., Carzino, R., Bianchini, P., Athanassiou, A. (2016). Polylactic Acid-Lemongrass Essential Oil Nanocapsules With Antimicrobial Properties. Pharmaceuticals, 9, 42.

7. Tcharkhtchi, A., Abdallah-Elhirtsi, S., Ebrahimi, K., Fitoussi, J., Shirinbayan, M., Farzaneh, S. (2014). Some New Concepts Of Shape Memory Effect Of Polymers. Polymers, 6, 1144-1163.

8. Hamad, K., Kaseem, M., Yang, H.W., Deri, F., Y. G. Ko. (2015). Properties And Medical Applications Of Polylactic Acid: A Review. Express Polymer Letters Vol.9, No.5,435-455

9. Rasal, R.M., Janorkar, A.V., Hirt, D.E. (2010). Poly Lactic Acid Modifications. Prog.Polym. Sci., 35, 338-356.

10. Dedenaro, G., Costa, S., Rugiero, I., Pedrini, P., Tamburini, E., Valorization Of Agri-Food Waste Via Fermentation: Production Of Lactic Acid As A Building Block For The Synthesis Of Biopolymers, Appl. Sci, 6, 379.(2016).

11. Danhier, F., Ansorena, E., Silva, J.M., Coco, R., Le Breton, A., Préat, V. (2012).Plga-Based Nano particles: An Overview Of Biomedical Applications. J. Control. Release, 161, 505-522.

12. Aumnate, C., Pongwisuthiruchte, A., Pattananuwat, P,. Potiyaraj,P. (2018). Fabrication Of Abs/Graphene Oxide Composite Filament For Fused Filament Fabrication (FFF) 3d Printing; Advances In Materials Science And Engineering. 
13. Zhang, Y., Nayak, T.R., Hong, H., Cai,W. (2012). Graphene: A Versatile Nanoplatform For Biomedical Applications.Nanoscale, 4, 3833-3842.

14. Bitounis, D., Ali-Boucetta, H., Hong, B.H., Min, D.H., Kostarelos, K. (2013). Prospects And Challenges Of Graphene In Biomedical Applications. Adv. Mater. 25, 2258-2268.

15. Paul, W., Sharma, C.P., (2011). Blood Compatibility And Biomedical Applications Of Graphene. Trends Biomater. Artif. Organs, 25, 91-94.

16. Xianfeng Zhou, Feng Liang, (2014). Application Of Graphene/Graphene Oxide In Biomedicine And Biotechnology; Current Medicinal Chemistry • November 2013, 21, 855-869 .

17. Sithiprumnea Du, Luca Fambri, Alessandro Pegoretti, (2016). Fused Deposition Modelling With Abs-Graphene Nanocomposites; Composites: Part A 85. 181-191

18. Wang, Y., Li, Z., Wang, J., Li, J., Lin, Y. (2011). Graphene And Graphene Oxide: Biofunctionalization And Applicationsin Biotechnology. Trends Biotechnol., 29, 205-212.

19. Scaffaro, R., Botta, L., Maio, A., Mistretta, M.C.; La Mantia, F.P. (2016) Effect Of Graphenenanoplatelets On The Physical And Antimicrobial Properties Of Biopolymer-Based Nanocomposites. 9, 351.

20. He Shen, Liming Zhang, Min Liu, Zhijun Zhang, (2012). Biomedical Applications Of Graphene; Theranostics, 2(3):283-294

21. Yong Cheol Shin, Su-Jin Song, Suck Won Hong, Seung Jo Jeong, Wojciech Chrzanowski, Jae Chang Lee, Dong-Wook Han, (2017). Multifaceted Biomedical Applications Of Functional Graphene Nanomaterials To Coated Substrates, Patterned Arrays And Hybrid Scaffolds; Nanomaterials, 7, 369

22. Shen, H., Zhang, L., Liu, M., Zhang, Z., Biomedical Applications Of Graphene. Theranostics 2012, 2, 283-294.

23. Zhang, D., Chi, B., Li, B., "Fabrication Of Highly Conductive Graphene Flexible Circuits By 3d Printing," Synthetic Metals, Vol. 217, Pp. 79-86, 2016.

24. Dul, S., Fambri, L., Pegoretti, A., "Fused Deposition Modelling With Abs - Graphene nano composites," Composites Part A: Applied Science And Manufacturing, Vol. 85, Pp. 181-191, 2016.

25. Mallick, P. K., Fiber reinforced Composites: Materials, Manufacturing, And Design, Second Edition, Marcel Dekker, Inc, 1993.

26. Matsuzaki, R., Ueda, M., Namiki, M., Jeong, T.K., Asahara, H., Horiguchi, K., Nakamura, T., Todoroki, A., Hirano, Y., Three-Dimensional Printing Of Continuous-Fiber Composites By In-Nozzle Impregnation. Sci. Rep. 2016, 6, 23058.

27. Rangisetty, S., Peel, L.D., The Effect Of Infill Patterns And Annealing On Mechanical Properties Of Additively Manufactured Thermoplastic Composites (2017) Asme 2017 Conference On Smart Materials, Adaptive Structures And Intelligent Systems, Smasis 2017,

28. Hsiao, W.-K., Lorber, B., Reitsamer, H., Khinast, J., 3d Printing Of Oral Drugs: A New Reality Or Hype, Expert Opin. Drug Deliv. 2018, 15.

29. Diegel, O., Xu, W., Potgieter, J., A Case Study Of Rapid Prototype As Design In Educational Engineering Projects. Int. J. Eng. Educ. 2006, 22, 350.

30. Khoo, Z.X., Teoh, J.E.M., Liu, Y., Chua, C.K., Yang, S., An, J., Leong, K.F., Yeong,W.Y., 3d Printing Of Smart Materials: A Review On Recent Progresses In 4d Printing. Virtual Phys. Prototyp. 2015, 10, 103-122.

31. Campbell, I., Bourell, D., Gibson, I., Additive Manufacturing: Rapid Prototyping Comes Of Age. Rapid Prototyp. J. 2012, 18, 255-258.

32. Umaras, E., Tsuzuki, M.S.G., Additive Manufacturing - Considerations On Geometric Accuracy And Factors Of Influence. Ifac-Papersonline 2017, 50, 14940-14945.

33. Loh, G.H., Pei, E., Harrison, D., Monzón, M.D., An Overview Of Functionally Graded Additive Manufacturing. Addit. Manuf. 2018, 23, 34-44.

34. Przybytek, A., Guba Nska, I., Kuci Nska Lipka, J., Janik, H., Polyurethanes As A Potential Medical-Grade Filament For Use In Fused Deposition Modeling 3d Printers-A Brief Review. Fibres Text. Eastern Eur. 2018, $6,120-125$.

35. Tsai, K.J., Dixon, S., Hale, L.R., Darbyshire, A., Martin, D., De Mel, A., Biomimetic Heterogenous Elastic Tissue Development. Npj Regen. Med. 2017, 2, 16.

36. Zocca, A., Colombo, P., Gomes, C.M., Günster, J., Additive Manufacturing Of Ceramics: Issues, Potentialities, And Opportunities. J. Am. Ceram. Soc. 2015, 98, 1983-2001.

37. Khatri, B., Lappe, K., Noetzel, D., Pursche, K., Hanemann, T., A 3d-Printable Polymer-Metal Soft-Magnetic Functional Composite-Development And Characterization. Materials 2018, 11, 189.

38. Hohimer, C.J., Petrossian, G., Ameli, A., Mo, C., Pötschke, P., Electrical Conductivity And Piezoresistive Response Of 3d Printed Thermoplastic Polyurethane/Multiwalled Carbon Nanotube Composites. In Behavior 
And Mechanics Of Multifunctional Materials And Composites Xii; International Society For Optics And Photonics: Bellingham, Wa, Usa, 2018; Volume 10596, P. 105960j.

39. Rumley-Ouellette, B.J., Wahry, J.H., Baker, A.M., Bernardin, J.D., Marchi, A.N., Todd, M.D., In Situ Printing Of Conductive Poly Lactic Acid Strain Sensors Embedded Into Additively Manufactured Parts. Struct. Health Monit. 2017.

40. Pranzo, D., Larizza, P., Filippini, D., Percoco, G., Extrusion-Based 3d Printing Of Microfluidic Devices For Chemical And Biomedical Applications: A Topical Review. Micromachines 2018, 9, 374.

41. Tanikella, N.G., Wittbrodt, B., Pearce, J.M., Tensile Strength Of Commercial Polymer Materials For Fused Filament Fabrication 3d Printing. Addit. Manuf. 2017, 15, 40-47.

42. Wu, W., Geng, P., Li, G., Zhao, D., Zhang, H., Zhao, J., Influence Of Layer Thickness And Raster Angle On The Mechanical Properties Of 3d-Printed Peek And A Comparative Mechanical Study Between Peek And Abs. Materials 2015, 8, 5834-5846,60. 